\title{
Free Vibration of Single Walled Carbon Nanotube Resting on Exponentially Varying Elastic Foundation
}

https://doi.org/10.1515/cls-2018-0019

Received May 28, 2018; accepted Jun 21, 2018

Abstract: In this article, free vibration of Single Walled Carbon Nanotube (SWCNT) resting on exponentially varying Winkler elastic foundation is investigated by using Differential Quadrature Method (DQM). Euler-Bernoulli beam theory is considered in conjunction with the nonlocal elasticity theory of Eringen. Step by step procedure is included and MATLAB code has been developed to obtain the numerical results for different scaling parameters as well as for four types of edge conditions. Obtained results are validated with known results in special cases showing good agreement. Further, numerical as well as graphical results are illustrated to show the effects of nonuniform parameter, nonlocal parameter, aspect ratio, Winkler modulus parameter and edge conditions on the frequency parameters.

Keywords: SWCNT; Euler-Bernoulli beam theory; DQM; Vibration; Exponentially Varying Elastic Foundation

\section{Introduction}

Application of nanomaterials has expanded in the area of physics, chemistry, engineering and nanotechnology because of their special properties like mechanical, electrical and electronic [1]. As a result of these properties, nanomaterials play very significant roles in various nanomechanical systems and nanomaterials. These materials include nanowires, nanoparticles, nanotubes, nanotube resonators [2], nanoactuators [3] etc. Various applications concerning CNT reinforced structure like reinforced beam, plate etc. can be found in the literatures [4-7]. one may also get detail information about nanobeams and

\footnotetext{
*Corresponding Author: Snehashish Chakraverty: Department of Mathematics, National Institute of Technology, Rourkela-769008, India; Email: sne_chak@yahoo.com

Subrat Kumar Jena: Department of Mathematics, National Institute of Technology, Rourkela-769008, India; Email: sjena430@gmail.com
}

nanoplates in the book [8]. Among these nanostructures, single walled carbon nanotubes viz. nanobeams attract more attention due to their great potential in engineering applications. So, one must have appropriate knowledge about the mechanical behaviors of nanobeams for accurate prediction of vibration characteristics. So far, many researchers have been developed various nonclassical continuum theories such as couple stress theory, strain gradient theory, micropolar theory and nonlocal elasticity theory. Out of these nonclassical continuum theories, nonlocal elasticity theory which was developed by Eringen [9], has been extensively used in the vibration of nanobeams.

During the past years, various elastic foundation models such as Winkler, Pasternak and Flonenko -Borodich foundations have been introduced. It is worth mentioning that the Winkler foundation model has been suggested in 1867 by Winkler which has become one of the fundamental scenario in this regard. Linear algebraic relationship between the normal displacement and the contact pressure [10] is assumed in the modeling of this foundation. This assumption makes it simple to get the closed-form solutions [11, 12]. On the other hand, it also gets a nonlinear behavior with less computations in comparison with other methods [13-17]. One may see different studies on Winkler elastic foundation in important papers viz. [18, 19]. Finite and infinite beams on a Winkler support have been addressed by Auersch [20]. As regards, Eisenberger and Clastornik [21] studied vibration and buckling of a beam resting on variable Winkler elastic foundation. Also, dynamic behavior of infinite beam has been investigated by Ruge and Birk [22]. Next, Lee [23] studied dynamic response of a Timoshenko beam subject to a moving concentrated mass. Finite strip method has been used by Huang and Thambiratnam [24] to investigate deflection of plates having moving accelerated loads resting on Winkler foundation. Further, $\mathrm{Oz}$ and Pakdemirli [25] has also examined resonances of shallow beams with elastic foundations. Elastic foundations with Winkler-Pasternak problems may also be found in [26, 27]. It may be noted that structural problems with elastic foundation have also been solved 
by few other methods such as differential transform [28], Rayleigh-Ritz [29] and Harmonic DQ [30, 31].

For the first time Bellman and Casti [32] introduced Differential Quadrature (DQ) method in the year 1971. One may use this powerful technique for solving linear and nonlinear differential equations arising in various dynamic problems. Later, Bert et al. [33] used this method for solving dynamical problem arising in the field of structural dynamics. Since then, this method is applied by various researchers for solving different types of structural problems such as linear or nonlinear. Different authors introduced different procedures to use edge conditions in the DQ method. In this regard, for the first time Bert et al. [34] proposed $\delta$ technique to use edge conditions. In this case [35], one may observe that one edge condition is used at the boundary point where as other edge condition is at a distance $\delta$ from the boundary point. This $\delta$ technique may be suitable for c-c edge but this is not useful for S-S and S-C edge conditions. In order to eradicate the shortcomings of the above approach, Bert further presented a new technique in applying edge conditions which may be found in [36-40]. In this technique, one just need to implement one boundary condition numerically while the other edge condition can be obtained from the DQ weighting coefficient matrices. Similarly, Tornabene et al. surveyed several methods under the heading of strong formulation finite element method (SFEM) which can be found in [41].

Though, some of the studies have been done on Winkler elastic foundation, but to the best of the present authors' knowledge, the article provides first time the frequency parameters of SWCNTs resting on an exponentially varying Winkler elastic foundation. In this article, EulerBernoulli beam theory in conjunction with nonlocal elasticity theory has been considered to illustrate the effects of the nonuniform parameter, nonlocal parameter, aspect ratio, Winkler modulus parameter and edge condition on the frequency parameter.

\section{Review of nonlocal elastic theory}

Nonlocal stress tensor $\sigma$ at a given point $x$ in conjunction to nonlocal elasticity theory can be expressed as [42]

$$
\sigma(x)=\int_{V} K\left(\left|x^{\prime}-x\right|, \alpha\right) \tau d V\left(x^{\prime}\right)
$$

where $\tau$ is the classical stress tensor, $K\left(\left|x^{\prime}-x\right|, \alpha\right)$ the nonlocal modulus and $\left|x^{\prime}-x\right|$ the Euclidean distance. One may note that the volume integral is considered over the region $V$. Here $\alpha$ is the material constant and it depends on both external and internal characteristic lengths.

From Hooke's law, one may have

$$
\tau(x)=C(x): \varepsilon(x)
$$

where $C$ is the fourth order elasticity tensor, $\varepsilon$ is the classical strain tensor and : denotes double dot product.

It may be noted that Eq. (1) is the integral constitutive relation and it is very complicated to solve. Hence we need an equivalent form of this equation which may be expressed as [42]

$$
\left(1-\alpha^{2} L^{2} \nabla^{2}\right) \sigma=\tau, \quad \alpha=\frac{e_{0} a}{L}
$$

where $\nabla^{2}$ is the Laplace operator, $e_{0}$ is a material constant, $a$ is an internal characteristic length and $L$ is the external characteristic length of the nanostructure. Here $e_{0} a$ is the nonlocal parameter which shows scale effect in on the nano-structures.

\section{Formulation of non-local Euler-Bernoulli beam theory}

In this study, Euler-Bernoulli beam theory along with the nonlocal elasticity theory of Eringen [9] have been considered for the investigation. For this regard, one must have adequate knowledge about energies. Here we have considered $\mathrm{x}$ coordinate along the length of the beam and $\mathrm{z}$ coordinate from the mid-plane of the beam. One may express strain energy $U$ as

$$
U=\frac{1}{2} \int_{0}^{L} \int_{A} \sigma_{x x} \varepsilon_{x x} d A d x,
$$

where $\sigma_{x x}$ is the normal stress, $L$ is the length of the beam and $A$ is the cross-section area. The strain-displacement relation may be given as

$$
\varepsilon_{x x}=-z \frac{\partial^{2} w}{\partial x^{2}},
$$

where $\varepsilon_{x x}$ is the normal strain and $w$ is the deflection function. Substituting Eq. (5) in Eq. (4), one may obtain

$$
U=-\frac{1}{2} \int_{0}^{L} M \frac{\partial^{2} w}{\partial x^{2}} d x,
$$

where $M=\int_{A} z \sigma_{x x} d A$, is the bending moment. In this study, free harmonic motion is considered, i.e. $w=$ 
$w_{0}(x) \sin \omega t$, where $\omega$ is the natural frequency of vibration. Using free harmonic motion in Eq. (6), we may obtain the strain energy $U$ as

$$
U=-\frac{1}{2} \int_{0}^{L} M \frac{d^{2} w_{0}}{d x^{2}} d x
$$

The kinetic energy Tis given as

$$
T=\frac{1}{2} \int_{0}^{L} \rho A \omega^{2} w_{0}^{2} d x,
$$

where $\rho$ is the mass density and $A$ is the area of beams. Now the potential energy may be given as [43]

$$
V=\frac{1}{2} \int_{0}^{L} f_{e} w_{0} d x,
$$

where $f_{e}$ is the density of reaction force of elastic foundation and is defined as $f_{e}=k_{w} w_{0}$. Here $k_{w}$ denotes the Winkler modulus of the elastic medium. Now by using Hamilton's principle $\int_{0}^{t} \delta T-(\delta U+\delta V) d t=0$ and setting the co-efficient of $\delta w_{0}$ to zero, one may obtain the governing equation as

$$
\frac{d^{2} M}{d x^{2}}-k_{w} w_{0}=-\rho A \omega^{2} w_{0}
$$

Based on Eringen's nonlocal elasticity theory, nonlocal constitutive relation for EBT nanobeam may be expressed as

$$
M-\mu \frac{d^{2} M}{d x^{2}}=-E I \frac{d^{2} w_{0}}{d x^{2}}
$$

where $\mu$ is the nonlocal parameter which can be expressed as $\mu=\left(e_{0} a\right)^{2}$ with $e_{0}$ and $a$ denoting material constant and internal characteristic length respectively, $I$ is the second moment of area and $E$ is Young's modulus. By using Eq. (10) and Eq. (11), $M$ may be expressed as

$$
M=-E I \frac{d^{2} w_{0}}{d x^{2}}+\mu\left(-\rho A \omega^{2} w_{0}+k_{w} w_{0}\right)
$$

Using Eq. (12) in Eq. (10), one may obtain the governing equation in terms of displacement as

$$
\begin{aligned}
& E I \frac{d^{4} w_{0}}{d x^{4}}-\mu k_{w} \frac{d^{2} w_{0}}{d x^{2}}+\rho A \omega^{2} \mu \frac{d^{2} w_{0}}{d x^{2}}+k_{w} w_{0} \\
& =\rho A \omega^{2} w_{0}
\end{aligned}
$$

Eq. (13) may be rewritten as

$$
\begin{aligned}
& \frac{d^{4} w_{0}}{d x^{4}}-\frac{\mu k_{w}}{E I} \frac{d^{2} w_{0}}{d x^{2}}+\frac{\rho A \omega^{2} \mu}{E I} \frac{d^{2} w_{0}}{d x^{2}}+\frac{k_{w} w_{0}}{E I} \\
& =\frac{\rho A \omega^{2} w_{0}}{E I}
\end{aligned}
$$

Let us introduce the following non-dimensional terms

$$
X=\frac{X}{L}
$$

$W=\frac{W_{0}}{L}$

$\lambda^{2}=\frac{\rho A \omega^{2} L^{4}}{E I_{0}}=$ frequency parameter

$K=\frac{k_{w} L^{4}}{E I_{0}}=$ Winkler modulus parameter.

Now, the proposed exponential variation in Winkler elastic foundation has now been expressed as

$$
K=\left(K_{w}\right) e^{-\eta X}
$$

where $K_{w}$ is the Winkler modulus parameter and $\eta$ is a positive constant.

Using the above non-dimensional terms and Eq. (15) in Eq. (14), we obtain the nondimensionalized form of the governing differential equation as

$$
\begin{aligned}
& \frac{d^{4} W}{d X^{4}}-\frac{\mu}{L^{2}} K_{w} e^{-\eta X} \frac{d^{2} W}{d X^{2}}+K_{w} e^{-\eta X} W \\
& =\lambda^{2}\left(W-\frac{\mu}{L^{2}} \frac{d^{2} W}{d X^{2}}\right)
\end{aligned}
$$

Next we introduce an overview of the differential quadrature method.

\section{Differential Quadrature Method}

In this present investigation, Quan and Chang's [44] approach is taken into consideration along with ChebyshevGauss-Lobatto grid points which is expressed as

$X_{i}=\frac{1}{2}\left[1-\cos \left(\frac{i-1}{N-1} \cdot \pi\right)\right]$.

It may also note that this is a non-uniform grid points.

One may express the derivatives of displacement function $W(X)$ at a given discrete point $i$ as [45]

$$
\begin{aligned}
W_{i}^{\prime} & =\sum_{j=1}^{N} A_{i j} W_{j} \\
W_{i}^{\prime \prime} & =\sum_{j=1}^{N} B_{i j} W_{j} \\
W_{i}^{\prime \prime \prime} & =\sum_{j=1}^{N} C_{i j} W_{j} \\
W_{i}^{I V} & =\sum_{j=1}^{N} D_{i j} W_{j}
\end{aligned}
$$


where $i=1,2, \ldots, N$ and $N$ is the number of discrete grid points.

Here $A_{i j}, B_{i j}, C_{i j}$ and $D_{i j}$ are the weighting coefficients of the first, second, third and fourth derivatives respectively.

\subsection{Determination of weighting coefficients}

Computation of weighting coefficient matrix $A=\left(A_{i j}\right)$ plays very significant role in DQ method. As per Quan and Chang's approach, matrix $A=\left(A_{i j}\right)$ can be computed by the following procedure.

For $i \neq j$

$$
\begin{gathered}
A_{i j}=\frac{1}{X_{j}-X_{i}} \prod_{\substack{k \neq i \\
k \neq j \\
k=1}}^{N} \frac{X_{i}-X_{k}}{X_{j}-X_{k}} \quad i=1,2, \ldots, N \\
\quad j=1,2, \ldots, N
\end{gathered}
$$

for $i=j$

$$
A_{i i}=\sum_{\substack{k \neq i \\ k=1}}^{N} \frac{1}{X_{i}-X_{k}} \quad i=1,2, \ldots, N .
$$

After weighting coefficients of first order derivatives are computed, one may easily get the weighting coefficients of higher order derivatives by simply matrix multiplication which is given as follows.

$$
\begin{gathered}
B=B_{i j}=\sum_{k=1}^{N} A_{i k} A_{k j} \\
C=C_{i j}=\sum_{k=1}^{N} A_{i k} B_{k j} \\
D=D_{i j}=\sum_{k=1}^{N} A_{i k} C_{k j}=\sum_{k=1}^{N} B_{i k} B_{k j} .
\end{gathered}
$$

\subsection{Application of boundary conditions}

Four classical boundary conditions such as SS, CS, CC and $C F$ are taken it consideration in the present study where the letters $S, C$, and $F$ denote simply supported, clamped and free edge conditions respectively.

Let us now denote

$$
\bar{A}_{1}=\left[\begin{array}{cccc}
0 & A_{1,2} & \cdots & A_{1, N} \\
0 & A_{2,2} & \cdots & A_{2, N} \\
\cdots & \cdots & \cdots & \cdots \\
0 & A_{N, 2} & \cdots & A_{N, N}
\end{array}\right]
$$

$$
\bar{A}_{2}=\left[\begin{array}{ccccc}
A_{1,1} & A_{1,2} & \cdots & A_{1, N-1} & 0 \\
A_{2,1} & A_{2,2} & \cdots & A_{2, N-1} & 0 \\
\cdots & \cdots & \cdots & \cdots & \cdots \\
A_{N, 1} & A_{N, 2} & \cdots & A_{N-1, N-1} & 0
\end{array}\right] .
$$

Now, the weighting coefficients of higher order derivatives for different edge conditions are given as below.

\section{Simply supported-simply supported}

$$
\begin{aligned}
\left\{W^{\prime}\right\} & =[\bar{A}]\{W\} . \\
\left\{W^{\prime \prime}\right\} & =[A][\bar{A}]\{W\}=[\bar{B}]\{W\} \text { with } \bar{B}=[A][\bar{A}] . \\
\left\{W^{\prime \prime \prime}\right\} & =[\bar{A}]\left\{W^{\prime \prime \prime}\right\}=[\bar{A}][\bar{B}]\{W\}=[\bar{C}]\{W\} . \\
\left\{W^{I V}\right\} & =[A]\left\{W^{\prime \prime \prime}\right\}=[A][\bar{C}]\{W\}=[\bar{B}][\bar{B}]\{W\} \\
& =[\bar{D}]\{W\}
\end{aligned}
$$

where $[\bar{D}]=[\bar{B}][\bar{B}]$ or $[\bar{D}]=[A][\bar{C}]$.

\section{Clamped-simply supported:}

$$
\begin{aligned}
\left\{W^{\prime}\right\} & =[\bar{A}]\{W\} \\
\left\{W^{\prime \prime}\right\} & =\left[\bar{A}_{1}\right]\left\{W^{\prime}\right\}=\left[\bar{A}_{1}\right][\bar{A}]\{W\} \\
& =[\bar{B}]\{W\} \text { with }[\bar{B}]=\left[\bar{A}_{1}\right][\bar{A}] . \\
\left\{W^{\prime \prime \prime}\right\} & =\left[\bar{A}_{2}\right]\left\{W^{\prime \prime}\right\}=\left[\bar{A}_{2}\right][\bar{B}]\{W\} \\
& =[\bar{C}]\{W\} \text { with }[\bar{C}]=\left[\bar{A}_{2}\right][\bar{B}] . \\
\left\{W^{I V}\right\} & =[A]\left\{W^{\prime \prime \prime}\right\}=[A][\bar{C}]\{W\} \\
& =[\bar{D}]\{W\} \text { with }[\bar{D}]=[A][\bar{C}] .
\end{aligned}
$$

\section{Clamped-Clamped:}

$$
\begin{aligned}
\left\{W^{\prime}\right\} & =[\bar{A}]\{W\} \\
\left\{W^{\prime \prime}\right\} & =[\bar{A}]\left\{W^{\prime}\right\}=[\bar{A}][\bar{A}]\{W\} \\
& =[\bar{B}]\{W\} \text { with }[\bar{B}]=[\bar{A}][\bar{A}] . \\
\left\{W^{\prime \prime \prime}\right\} & =[A]\left\{W^{\prime \prime}\right\}=[A][\bar{B}]\{W\} \\
& =[\bar{C}]\{W\} \text { with }[\bar{C}]=[A][\bar{B}] . \\
\left\{W^{I V}\right\} & =[A]\left\{W^{\prime \prime \prime}\right\}=[A][\bar{C}]\{W\} \\
& =[\bar{D}]\{W\} \text { with }[\bar{D}]=[A][\bar{C}] .
\end{aligned}
$$

\section{Clamped-free:}

$$
\begin{aligned}
& \left\{W^{\prime}\right\}=\left[\bar{A}_{1}\right]\{W\} \\
& \left\{W^{\prime \prime}\right\}=\left[\bar{A}_{1}\right]\left\{W^{\prime}\right\}=\left[\bar{A}_{1}\right]\left[\bar{A}_{1}\right]\{W\}
\end{aligned}
$$


Table 1: Comparisons of frequency parameter $(\sqrt{\lambda})$ for SS case

\begin{tabular}{cccccccccc}
\hline \multirow{2}{*}{$K_{w}$} & \multicolumn{3}{c}{$\sqrt{\lambda_{1}}$} & \multicolumn{3}{c}{$\sqrt{\lambda_{2}}$} & & $\sqrt{\lambda_{3}}$ \\
\cline { 2 - 9 } & Present & Ref. [28] & Ref. [18] & Present & Ref. [28] & Ref. [18] & Present & Ref. [28] & Ref. [18] \\
\hline 10 & 3.2192 & 3.2192 & 3.2192 & 6.2932 & 6.2932 & 6.2932 & 9.4277 & 9.4277 & 9.4277 \\
50 & 3.4844 & 3.4844 & 3.4844 & 6.3329 & 6.3329 & 6.3329 & 9.4396 & 9.4396 & 9.4396 \\
100 & 3.7483 & 3.7483 & 3.7483 & 6.3816 & 6.3816 & 6.3816 & 9.4544 & 9.4544 & 9.4544 \\
200 & 4.1527 & 4.1527 & 4.1527 & 6.4757 & 6.4757 & 6.4757 & 9.4839 & 9.4839 & 9.4839 \\
500 & 4.9438 & 4.9438 & 4.9438 & 6.7358 & 6.7358 & 6.7358 & 9.5706 & 9.5706 & 9.5706 \\
1000 & 5.7556 & 5.7556 & 5.7556 & 7.1121 & 7.1121 & 7.1121 & 9.7101 & 9.7101 & 9.7101 \\
1500 & 6.3219 & 6.3219 & 6.3219 & 7.4366 & 7.4366 & 7.4366 & 9.8439 & 9.8439 & 9.8439 \\
2000 & 6.7673 & 6.7673 & 6.7673 & 7.7235 & 7.7235 & 7.7235 & 9.9724 & 9.9724 & 9.9724 \\
\hline
\end{tabular}

$$
\begin{aligned}
& =[\bar{B}]\{W\} \text { with }[\bar{B}]=\left[\bar{A}_{1}\right]\left[\bar{A}_{1}\right] . \\
\left\{W^{\prime \prime \prime}\right\} & =\left[\bar{A}_{2}\right]\left\{W^{\prime \prime}\right\}=\left[\bar{A}_{2}\right][\bar{B}]\{W\} \\
& =[\bar{C}]\{W\} \text { with }[\bar{C}]=\left[\bar{A}_{2}\right][\bar{B}] . \\
\left\{W^{I V}\right\} & =\left[\bar{A}_{2}\right]\left\{W^{\prime \prime \prime}\right\}=\left[\bar{A}_{2}\right][\bar{C}]\{W\} \\
& =[\bar{D}]\{W\} \text { with }[\bar{D}]=\left[\bar{A}_{2}\right][\bar{C}] .
\end{aligned}
$$

Substituting the value of Eq. (17) into Eq. (16), one may obtain generalized eigenvalue problem as

$$
[S]\{W\}=\lambda^{2}[T]\{W\}
$$

where $S$ is the stiffness matrix and $T$ is the mass matrix.

\section{Numerical results and discussions}

Eq. (23) is solved by using MATLAB program which is developed by the authors and frequency parameters $\sqrt{\lambda}$ have been obtained. DQ method has been implemented properly along with the boundary conditions in the coefficient matrix. Following parameters are taken from [46] for computational purpose.

$E=30 \times 10^{6} \mathrm{~Pa}, L=10 \mathrm{~nm}$, Poisson's ratio $(v)=0.3$, $K_{s}=\frac{5}{6}$ and unless mentioned $\frac{L}{h}=10$.

\subsection{Validation}

Validation of the present method is done in two ways. Firstly, we consider Euler-Bernoulli beam with nonlocal parameter $\mu=0$ and non-uniform parameter $\eta=0$. we compare our results of frequency parameter $(\sqrt{\lambda})$ with those available in the literature $[18,28]$ for different Winkler modulus parameter and are given in Tables 1-3 . Secondly, we consider a nanobeam not resting on any elastic
Table 2: Comparisons of frequency parameter $(\sqrt{\lambda})$ for CC case

\begin{tabular}{ccccccc}
\hline \multirow{2}{*}{$K_{w}$} & \multicolumn{2}{c}{$\sqrt{\lambda_{1}}$} & \multicolumn{2}{c}{$\sqrt{\lambda_{2}}$} & \multicolumn{2}{c}{$\sqrt{\lambda_{3}}$} \\
\cline { 2 - 7 } & Present & Ref. [28] & Present & Ref. [28] & Present & Ref. [28] \\
\hline 1 & 4.7324 & 4.7324 & 7.8537 & 7.8537 & 10.9958 & 10.9958 \\
10 & 4.7534 & 4.7534 & 7.8583 & 7.8583 & 10.9975 & 10.9975 \\
100 & 4.9503 & 4.9503 & 7.9043 & 7.9043 & 11.0144 & 11.0144 \\
1000 & 6.2239 & 6.2239 & 8.3251 & 8.3251 & 11.1790 & 11.1790 \\
\hline
\end{tabular}

Table 3: Comparisons of frequency parameter $(\sqrt{\lambda})$ for CF case

\begin{tabular}{ccccccc}
\hline \multirow{2}{*}{$K_{w}$} & \multicolumn{2}{c}{$\sqrt{\lambda_{1}}$} & \multicolumn{2}{c}{$\sqrt{\lambda_{2}}$} & \multicolumn{2}{c}{$\sqrt{\lambda_{3}}$} \\
\cline { 2 - 7 } & Present & Ref. [28] & Present & Ref. [28] & Present & Ref. [28] \\
\hline 1 & 1.9119 & 1.9119 & 4.6965 & 4.6965 & 7.8552 & 7.8552 \\
10 & 2.1746 & 2.1746 & 4.7180 & 4.7180 & 7.8599 & 7.8599 \\
100 & 3.2557 & 3.2557 & 4.9191 & 4.9191 & 7.9058 & 7.9058 \\
1000 & 5.6407 & 5.6407 & 6.2082 & 6.2082 & 8.3264 & 8.3264 \\
\hline
\end{tabular}

foundation viz. $\eta=0$ and . our results of fundamental frequency parameter $(\lambda)$ are now compared with Reddy [46], Aydogu [47] and Eltaher [48] for different nonlocal parameters $(\mu)$ which are presented in Tables 4-5. In Table 4, aspect ratio $(L / h)$ is taken as 10 . Similarly, in Table 5 , fundamental frequency parameter $(\lambda)$ for SS nanobeam is compared with Aydogu [47] and Eltaher [48] with aspect ratio $(L / h)$ as 20 . From these Tables $1-5$, one may observe close agreement of results with those available in the literatures.

\subsection{Convergence}

Minimum number of grid points have been obtained by studying convergence to obtain the final results. To show how the solution is affected by the grid points, variations of the frequency parameters $(\sqrt{\lambda})$ with the number of grid points are shown in Figure 1 and in Tables 6-9 for SS,CS,CC 
(a) Convergence of SS nanobeam

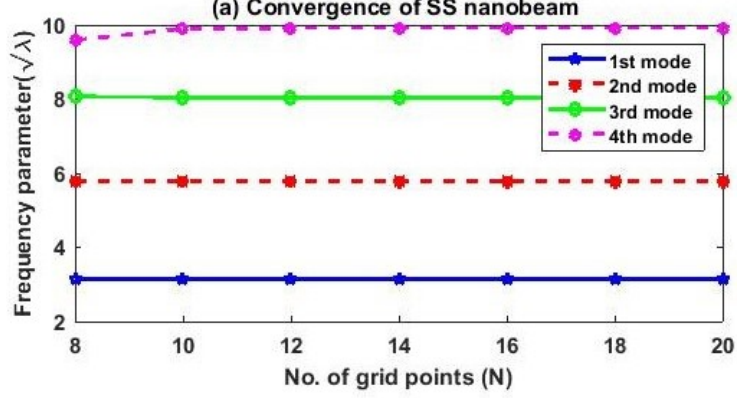

(c) Convergence of $\mathrm{CC}$ nanobeam

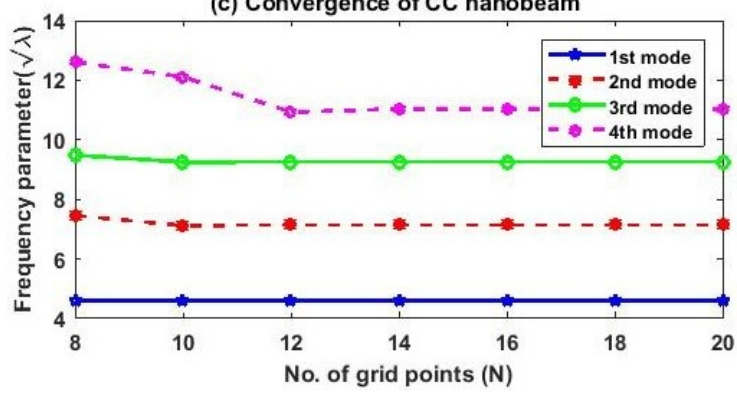

(b) Convergence of CS nanobeam

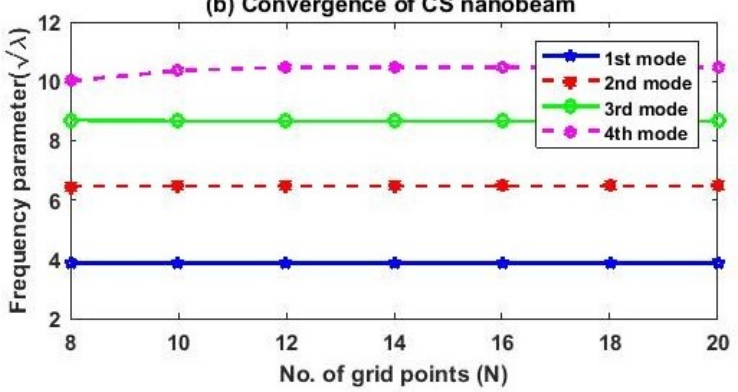

(d) Convergence of CF nanobeam

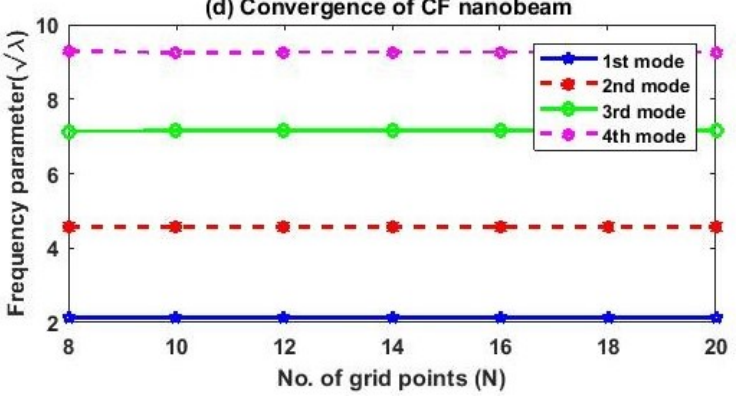

Figure 1: Variation of frequency parameters with number of terms for SS, CS, CC and CF conditions

Table 4: Comparisons of first fundamental frequency parameter $(\lambda)$ for SS Nano beam

\begin{tabular}{ccccc}
\hline$\mu$ & Present & Reddy [46] & Aydogu [47] & Eltaher [48] \\
\hline 0 & 9.8696 & 9.8696 & 9.8696 & 9.8696 \\
1 & 9.4159 & 9.4159 & 9.4124 & 9.4159 \\
2 & 9.0195 & 9.0195 & 9.0133 & 9.0195 \\
3 & 8.6693 & 8.6693 & 8.6611 & 8.6693 \\
4 & 8.3569 & 8.3569 & 8.3472 & 8.3569 \\
\hline
\end{tabular}

Table 5: Comparisons of first fundamental frequency parameter $(\lambda)$ for SS Nano beam

\begin{tabular}{cccc}
\hline$\mu$ & Aydogu [47] & Eltaher [48] & Present \\
\hline 0 & 9.8696 & 9.8798 & 9.8696 \\
1 & 9.7498 & 9.4238 & 9.7500 \\
2 & 9.6343 & 9.0257 & 9.63473 \\
3 & 9.5228 & 8.6741 & 9.5234 \\
4 & 9.4150 & 8.3606 & 9.4158 \\
\hline
\end{tabular}

and CF cases. Here, we have considered $L=10, \eta=$ $0.2, \mu=1$ and $K_{w}=10$. The convergence is shown for SS, $\mathrm{CS}, \mathrm{CC}$ and CF conditions. From this figure, one may note that with increase in number of grid points, the convergence is achieving fast. One may also observe that twelve grid points are sufficient to compute the desired results.

\subsection{Effect of nonlocal parameter}

In this subsection, first four frequency parameters $(\sqrt{\lambda})$ of nanobeam are obtained for different nonlocal parameters. We have taken the values of nonlocal parameter as $0,0.2,0.4,0.6,0.8,1 \mathrm{~nm}^{2}$. In this study, all the classical boundary conditions such as SS, CS, CC and CF are considered for investigation. Both tabular and graphical results are depicted by taking other parameters such as nonuniform parameter $(\eta)$ as 0.5 , Winkler modulus parameter $K_{W}=50$ and $L=10 \mathrm{~nm}$. First four frequency parameters of SS, CS, CC and CF edges for different nonlocal parameters are presented Tables 10-13. From these tables, one may observe that frequency parameters decrease with increase in nonlocal parameter except first fundamental frequency parameter of CF nanobeams. One may also notice that frequency parameters increase with increase in mode number. Here one important point to note that CC nanobeams are having highest frequency parameters than other set of boundary conditions. Figure 2 show variation of frequency ratio with nonlocal parameters for different edges such as SS, CS, CC and CF.

\subsection{Effect of nonuniform parameter}

Effect of non-uniform parameter $\eta$ on first four frequency parameters is analyzed by taking non-uniform parameter $\eta$ as $0,0.2,0.4,0.6,0.8$ and 1 . One may note that $\eta$ takes positive values only. Tables 14-17 and Figure 3 illustrate the 
Table 6: Frequency parameter $(\sqrt{\lambda})$ for SS case for different grid points

\begin{tabular}{ccccc}
\hline$N$ & $\sqrt{\lambda_{1}}$ & $\sqrt{\lambda_{2}}$ & $\sqrt{\lambda_{3}}$ & $\sqrt{\lambda_{4}}$ \\
\hline 8 & 3.137047387663272 & 5.792189727676567 & 8.069652183108396 & 9.587980174560933 \\
10 & 3.137043626122775 & 5.792222858427450 & 8.044736703926970 & 9.900828395069310 \\
12 & 3.137043645950507 & 5.792229270220762 & 8.043907944491545 & 9.917670536656008 \\
14 & 3.137043645870416 & 5.792229198324495 & 8.043924442382881 & 9.918214267400069 \\
16 & 3.137043645874682 & 5.792229199028369 & 8.043923930463707 & 9.918206815221069 \\
18 & 3.137043645875629 & 5.792229199022920 & 8.043923941298058 & 9.918207137096605 \\
20 & 3.137043645883244 & 5.792229199024242 & 8.043923941117477 & 9.918207128737370 \\
\hline
\end{tabular}

Table 7: $(\sqrt{\lambda})$ for CS case for different grid points

\begin{tabular}{ccccc}
\hline$N$ & $\sqrt{\lambda_{1}}$ & $\sqrt{\lambda_{2}}$ & $\sqrt{\lambda_{3}}$ & $\sqrt{\lambda_{4}}$ \\
\hline 8 & 3.856973271397841 & 6.463369136400239 & 8.698387847145867 & 10.013373349121141 \\
10 & 3.857068900732693 & 6.472653477925265 & 8.652930571005422 & 10.358046845755311 \\
12 & 3.857068049264370 & 6.472440609260722 & 8.655034716695576 & 10.473304478306719 \\
14 & 3.857068057194151 & 6.472445866438307 & 8.654843259979375 & 10.470245167091482 \\
16 & 3.857068057157146 & 6.472445777886075 & 8.654851649036990 & 10.470550548000791 \\
18 & 3.857068057156827 & 6.472445779054758 & 8.654851382273554 & 10.470535930174004 \\
20 & 3.857068057112573 & 6.472445779046844 & 8.654851388706513 & 10.470536499911651 \\
\hline
\end{tabular}

Table 8: Frequency parameter $(\sqrt{\lambda})$ for CC case for different grid points

\begin{tabular}{ccccc}
\hline$N$ & $\sqrt{\lambda_{1}}$ & $\sqrt{\lambda_{2}}$ & $\sqrt{\lambda_{3}}$ & $\sqrt{\lambda_{4}}$ \\
\hline 8 & 4.614872016555520 & 7.460588192516093 & 9.486498247290566 & 12.609873904602038 \\
10 & 4.615425147178145 & 7.121835740601477 & 9.253522431196084 & 12.111957988099142 \\
12 & 4.615417617988975 & 7.147480265640297 & 9.261700624304588 & 10.931617747978734 \\
14 & 4.615417714547347 & 7.145802024795920 & 9.260855837455789 & 11.037275335647557 \\
16 & 4.615417713845297 & 7.145867516572760 & 9.260896551844526 & 11.015322124620903 \\
18 & 4.615417713847023 & 7.145865717988663 & 9.260895055673364 & 11.017480223039190 \\
20 & 4.615417713848704 & 7.145865754113659 & 9.260895097840340 & 11.017322575498191 \\
\hline
\end{tabular}

Table 9: Frequency parameter $(\sqrt{\lambda})$ for CF case for different grid points

\begin{tabular}{ccccc}
\hline & $\sqrt{\lambda_{1}}$ & $\sqrt{\lambda_{2}}$ & $\sqrt{\lambda_{3}}$ & $\sqrt{\lambda_{4}}$ \\
\hline 8 & 2.131906821758265 & 4.568180334350084 & 7.135038616372697 & 9.294609304241696 \\
10 & 2.131906968161213 & 4.569107989776002 & 7.151439435905257 & 9.246361354117974 \\
12 & 2.131906968641838 & 4.569093872033319 & 7.151500240014390 & 9.260430132289168 \\
14 & 2.131906968623912 & 4.569094037626583 & 7.151498157051591 & 9.259396249267780 \\
16 & 2.131906968654128 & 4.569094036365876 & 7.151498264697817 & 9.259455049663973 \\
18 & 2.131906968600308 & 4.569094036328919 & 7.151498262389548 & 9.259452763225076 \\
20 & 2.131906968944130 & 4.569094036409303 & 7.151498262428689 & 9.259452830507044 \\
\hline
\end{tabular}



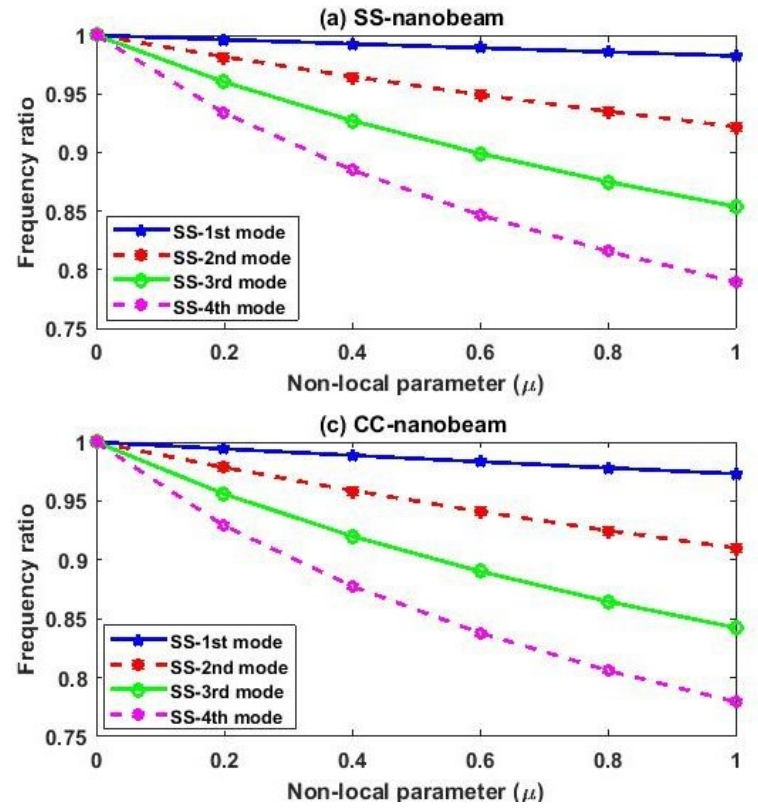

Figure 2: Variation of frequency parameter with nonlocal parameter

Table 10: Frequency parameter for SS case for different nonlocal parameter

\begin{tabular}{ccccc}
\hline$\mu$ & $\sqrt{\lambda_{1}}$ & $\sqrt{\lambda_{2}}$ & $\sqrt{\lambda_{3}}$ & $\sqrt{\lambda_{4}}$ \\
\hline 0 & 3.3618 & 6.3135 & 9.4338 & 12.5701 \\
0.2 & 3.3493 & 6.1970 & 9.0574 & 11.7377 \\
0.4 & 3.3372 & 6.0908 & 8.7463 & 11.1241 \\
0.6 & 3.3254 & 5.9935 & 8.4825 & 10.6438 \\
0.8 & 3.3139 & 5.9037 & 8.2545 & 10.2523 \\
1.0 & 3.3027 & 5.8205 & 8.0545 & 9.9238 \\
\hline
\end{tabular}

Table 11: Frequency parameter for CS case for different nonlocal parameter

\begin{tabular}{ccccc}
\hline$\mu$ & $\sqrt{\lambda_{1}}$ & $\sqrt{\lambda_{2}}$ & $\sqrt{\lambda_{3}}$ & $\sqrt{\lambda_{4}}$ \\
\hline 0 & 4.0462 & 7.0899 & 10.2172 & 13.3549 \\
0.2 & 4.0259 & 6.9473 & 9.7876 & 12.4373 \\
0.4 & 4.0061 & 6.8181 & 9.4358 & 11.7684 \\
0.6 & 3.9869 & 6.7004 & 9.1397 & 11.2487 \\
0.8 & 3.9682 & 6.5923 & 8.8854 & 10.8274 \\
1.0 & 3.9501 & 6.4927 & 8.6633 & 10.4753 \\
\hline
\end{tabular}

variation of frequency parameter with nonuniform parameter $\eta$ for all the classical boundary conditions such as SS, $\mathrm{CS}$, CC and CF. Here, computation is done with nonlocal parameter $\mu=1 \mathrm{~nm}^{2}, L=10 \mathrm{~nm}$ and $K_{w}=100$. It may also be noted from the graph that the frequency parameter decreases with increase in the non-uniform parameter and this decrease is more significant in case of higher modes.
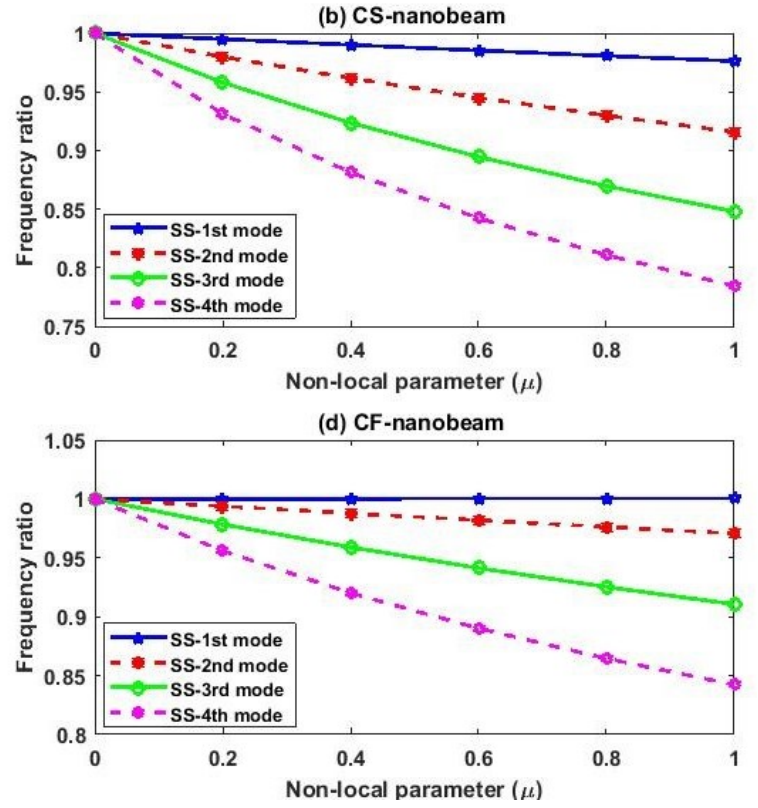

Table 12: Frequency parameter $(\sqrt{\lambda})$ for CC case for different nonlocal parameter

\begin{tabular}{ccccc}
\hline$\mu$ & $\sqrt{\lambda_{1}}$ & $\sqrt{\lambda_{2}}$ & $\sqrt{\lambda_{3}}$ & $\sqrt{\lambda_{4}}$ \\
\hline 0 & 4.8001 & 7.8688 & 11.0013 & 14.1398 \\
0.2 & 4.7726 & 7.6985 & 10.5166 & 13.1348 \\
0.4 & 4.7460 & 7.5450 & 10.1229 & 12.4094 \\
0.6 & 4.7202 & 7.4056 & 9.7937 & 11.8495 \\
0.8 & 4.6951 & 7.2781 & 9.5124 & 11.3976 \\
1.0 & 4.6707 & 7.1609 & 9.2678 & 11.0214 \\
\hline
\end{tabular}

Table 13: Frequency parameter $(\sqrt{\lambda})$ for CF case for different nonlocal parameter

\begin{tabular}{ccccc}
\hline$\mu$ & $\sqrt{\lambda_{1}}$ & $\sqrt{\lambda_{2}}$ & $\sqrt{\lambda_{3}}$ & $\sqrt{\lambda_{4}}$ \\
\hline 0 & 2.5561 & 4.7657 & 7.8703 & 11.0012 \\
0.2 & 2.5564 & 4.7363 & 7.7006 & 10.5164 \\
0.4 & 2.5567 & 4.7076 & 7.5478 & 10.1225 \\
0.6 & 2.5570 & 4.6797 & 7.4092 & 9.7931 \\
0.8 & 2.5573 & 4.6525 & 7.2827 & 9.5114 \\
1.0 & 2.5577 & 4.6260 & 7.1665 & 9.2664 \\
\hline
\end{tabular}

\subsection{Effect of length-to-height ratio}

In this subsection, effect of length-to-height ratio $(\mathrm{L} / \mathrm{h})$ on the first four frequency parameters has been analyzed for all the classical boundary conditions such as SS, CS, $\mathrm{CC}$ and CF. First four frequency parameters of nanobeam are given in Tables 18-21 for different $L / h(10,20,30,40$, 
(a) SS nanobeam

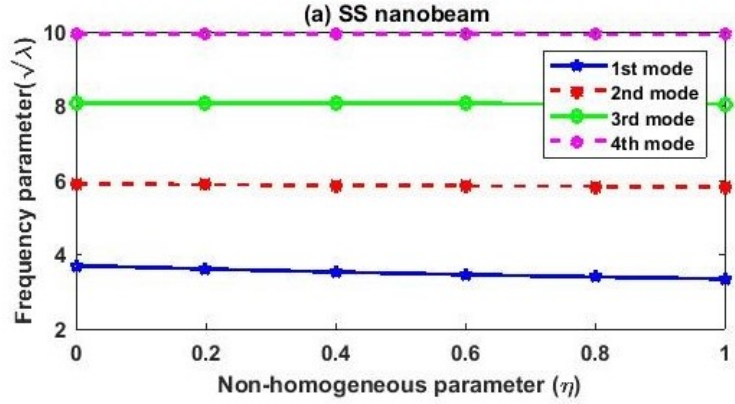

(c) CC nanobeam

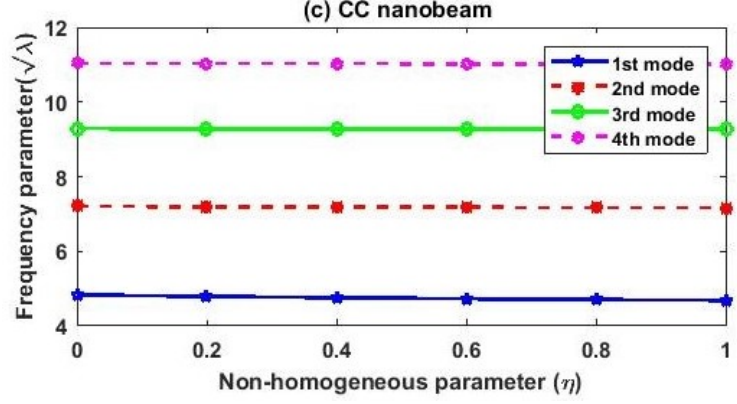

(b) CS nanobeam

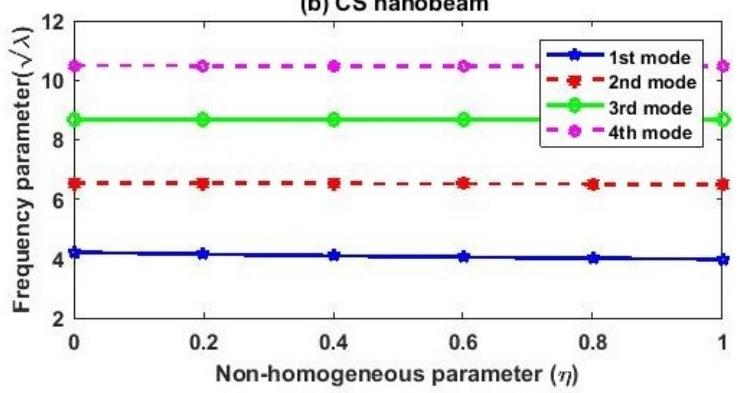

(d) CF nanobeam

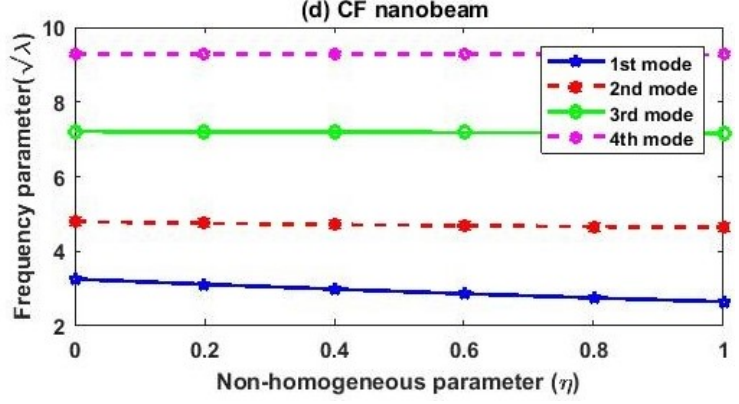

Figure 3: Variation of frequency parameter with non-uniform or non-homogeneous parameter

Table 14: Frequency parameter $(\sqrt{\lambda})$ for SS case for different nonuniform parameter

\begin{tabular}{ccccc}
\hline$\eta$ & $\sqrt{\lambda_{1}}$ & $\sqrt{\lambda_{2}}$ & $\sqrt{\lambda_{3}}$ & $\sqrt{\lambda_{4}}$ \\
\hline 0 & 3.7061 & 5.9068 & 8.0876 & 9.9416 \\
0.2 & 3.6136 & 5.8847 & 8.0790 & 9.9370 \\
0.4 & 3.5323 & 5.8664 & 8.0720 & 9.9332 \\
0.6 & 3.4613 & 5.8513 & 8.0662 & 9.9301 \\
0.8 & 3.3997 & 5.8389 & 8.0615 & 9.9276 \\
1.0 & 3.3466 & 5.8286 & 8.0576 & 9.9255 \\
\hline
\end{tabular}

Table 15: Frequency parameter $(\sqrt{\lambda})$ for CS case for different nonuniform parameter

\begin{tabular}{ccccc}
\hline$\eta$ & $\sqrt{\lambda_{1}}$ & $\sqrt{\lambda_{2}}$ & $\sqrt{\lambda_{3}}$ & $\sqrt{\lambda_{4}}$ \\
\hline 0 & 4.2066 & 6.5554 & 8.6900 & 10.4904 \\
0.2 & 4.1443 & 6.5393 & 8.6831 & 10.4865 \\
0.4 & 4.0912 & 6.5260 & 8.6774 & 10.4833 \\
0.6 & 4.0461 & 6.5150 & 8.6728 & 10.4806 \\
0.8 & 4.0080 & 6.5060 & 8.6689 & 10.4785 \\
1.0 & 3.9760 & 6.4986 & 8.6658 & 10.4767 \\
\hline
\end{tabular}

50). In this table, computation is done with $\mu=1 \mathrm{~nm}^{2}$, $K_{w}=50$ and $\eta=0.5$. Graphical results are presented in Figure 4,where variation of first four frequency parameters with $L / h$ has been shown. In this figure, varies from 10 to 50.
Table 16: Frequency parameter $(\sqrt{\lambda})$ for CC case for different nonuniform parameter

\begin{tabular}{ccccc}
\hline$\eta$ & $\sqrt{\lambda_{1}}$ & $\sqrt{\lambda_{2}}$ & $\sqrt{\lambda_{3}}$ & $\sqrt{\lambda_{4}}$ \\
\hline 0 & 4.8330 & 7.2079 & 9.2896 & 11.0344 \\
0.2 & 4.7923 & 7.1958 & 9.2840 & 11.0310 \\
0.4 & 4.7582 & 7.1858 & 9.2793 & 11.0283 \\
0.6 & 4.7298 & 7.1776 & 9.2755 & 11.0260 \\
0.8 & 4.7061 & 7.1709 & 9.2724 & 11.0241 \\
1.0 & 4.6864 & 7.1653 & 9.2698 & 11.0226 \\
\hline
\end{tabular}

Table 17: Frequency parameter $(\sqrt{\lambda})$ for CF case for different nonuniform parameter

\begin{tabular}{ccccc}
\hline$\eta$ & $\sqrt{\lambda_{1}}$ & $\sqrt{\lambda_{2}}$ & $\sqrt{\lambda_{3}}$ & $\sqrt{\lambda_{4}}$ \\
\hline 0 & 3.2565 & 4.7927 & 7.2134 & 9.2882 \\
0.2 & 3.1165 & 4.7510 & 7.2013 & 9.2825 \\
0.4 & 2.9860 & 4.7160 & 7.1913 & 9.2779 \\
0.6 & 2.8647 & 4.6868 & 7.1832 & 9.2741 \\
0.8 & 2.7525 & 4.6624 & 7.1764 & 9.2710 \\
1.0 & 2.6492 & 4.6422 & 7.1709 & 9.2684 \\
\hline
\end{tabular}

\subsection{Effect of boundary condition}

One need to have adequate knowledge about boundary conditions for designing any engineering structures. It helps designers to gather some important information without carrying out detail experimental investigation. Therefore, it is quite important to study effect of boundary 
(a) SS nanobeam

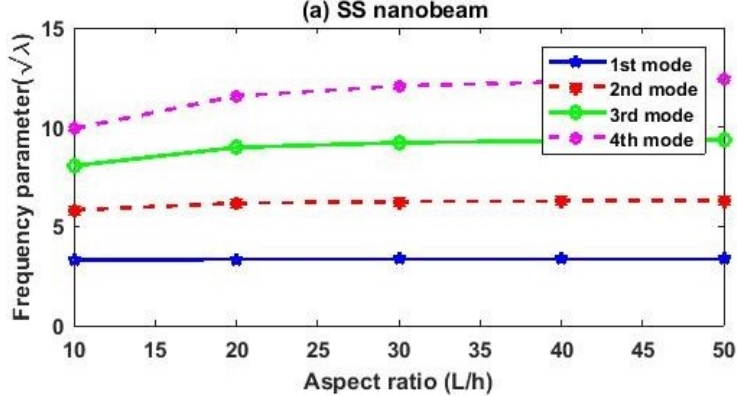

(c) CC nanobeam

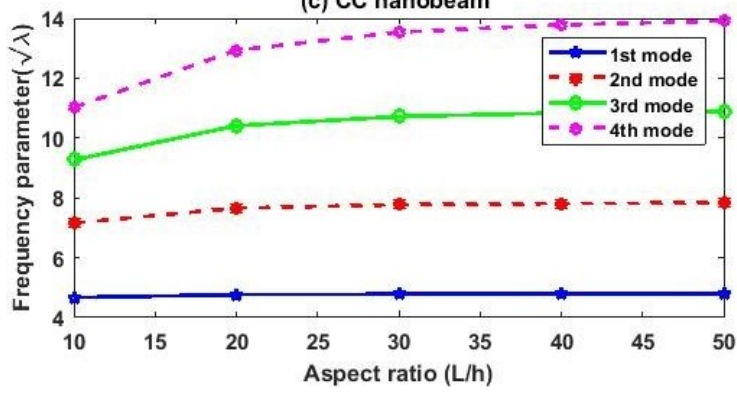

Figure 4: Variation of frequency parameter with length-to-height ratio

Table 18: Frequency parameter $(\sqrt{\lambda})$ for SS case for different length-to-height ratio

\begin{tabular}{ccccc}
\hline$L / h$ & $\sqrt{\lambda_{1}}$ & $\sqrt{\lambda_{2}}$ & $\sqrt{\lambda_{3}}$ & $\sqrt{\lambda_{4}}$ \\
\hline 10 & 3.3027 & 5.8205 & 8.0545 & 9.9238 \\
20 & 3.3463 & 6.1696 & 8.9744 & 11.5682 \\
30 & 3.3548 & 6.2474 & 9.2152 & 12.0729 \\
40 & 3.3579 & 6.2759 & 9.3077 & 12.2782 \\
50 & 3.3593 & 6.2893 & 9.3521 & 12.3794 \\
\hline
\end{tabular}

Table 19: Frequency parameter $(\sqrt{\lambda})$ for CS case for different length-to-height ratio

\begin{tabular}{ccccc}
\hline$L / h$ & $\sqrt{\lambda_{1}}$ & $\sqrt{\lambda_{2}}$ & $\sqrt{\lambda_{3}}$ & $\sqrt{\lambda_{4}}$ \\
\hline 10 & 3.9501 & 6.4927 & 8.6633 & 10.4753 \\
20 & 4.0209 & 6.9138 & 9.6934 & 12.2519 \\
30 & 4.0348 & 7.0088 & 9.9672 & 12.8053 \\
40 & 4.0398 & 7.0437 & 10.0728 & 13.0317 \\
50 & 4.0421 & 7.0602 & 10.1236 & 13.1435 \\
\hline
\end{tabular}

conditions on frequency parameter. So,the effect of boundary condition on the frequency parameter is studied in this subsection. Figure 5 illustrates variation of frequency parameter with nonlocal parameter for different boundary conditions. The values of parameters are taken as $L=$ $10 \mathrm{~nm}, K_{w}=50$ and $\eta=0.5$ for plotting the figure. One may observe from the figure that CC nanobeams are having highest frequency parameter whereas $\mathrm{CF}$ nanobeams possess lowest frequency parameter. It may also be noted

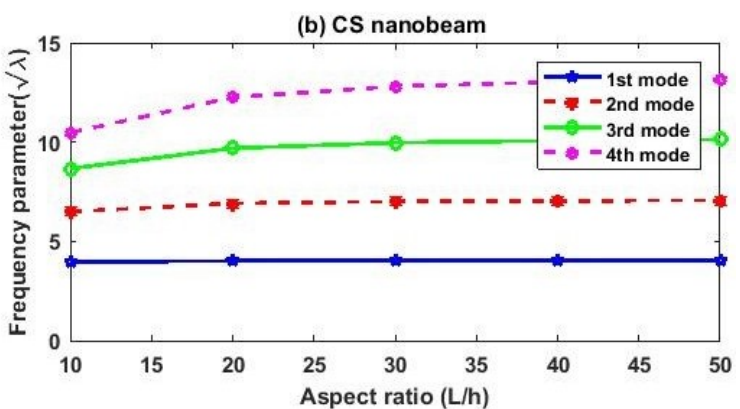

(d) CF nanobeam

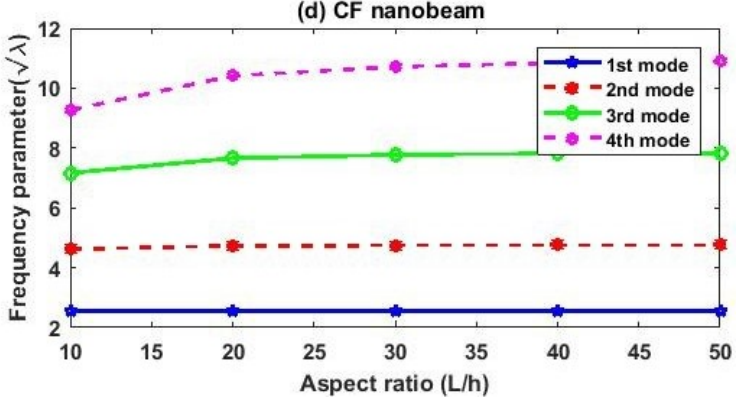

Table 20: Frequency parameter $(\sqrt{\lambda})$ for CC case for different length-to-height ratio

\begin{tabular}{ccccc}
\hline$L / h$ & $\sqrt{\lambda_{1}}$ & $\sqrt{\lambda_{2}}$ & $\sqrt{\lambda_{3}}$ & $\sqrt{\lambda_{4}}$ \\
\hline 10 & 4.6707 & 7.1609 & 9.2678 & 11.0214 \\
20 & 4.7659 & 7.6587 & 10.4109 & 12.9332 \\
30 & 4.7847 & 7.7719 & 10.7187 & 13.5365 \\
40 & 4.7914 & 7.8136 & 10.8378 & 13.7844 \\
50 & 4.7945 & 7.8332 & 10.8953 & 13.9072 \\
\hline
\end{tabular}

Table 21: Frequency parameter $(\sqrt{\lambda})$ for CF case for different length-to-height ratio

\begin{tabular}{ccccc}
\hline$L / h$ & $\sqrt{\lambda_{1}}$ & $\sqrt{\lambda_{2}}$ & $\sqrt{\lambda_{3}}$ & $\sqrt{\lambda_{4}}$ \\
\hline 10 & 2.5577 & 4.6260 & 7.1665 & 9.2664 \\
20 & 2.5565 & 4.7290 & 7.6610 & 10.4107 \\
30 & 2.5562 & 4.7492 & 7.7738 & 10.7186 \\
40 & 2.5561 & 4.7564 & 7.8153 & 10.8377 \\
50 & 2.5561 & 4.7597 & 7.8349 & 10.8952 \\
\hline
\end{tabular}

that frequency parameters decrease with increase in nonlocal parameter for SS, CS, and CC edge but in case of first mode of CF nanobeams, it is exactly opposite.

\subsection{Effect of Winkler modulus parameter}

Effect of Winkler modulus parameter $\left(K_{w}\right)$ on the first four frequency parameters has been studied. First four fre- 


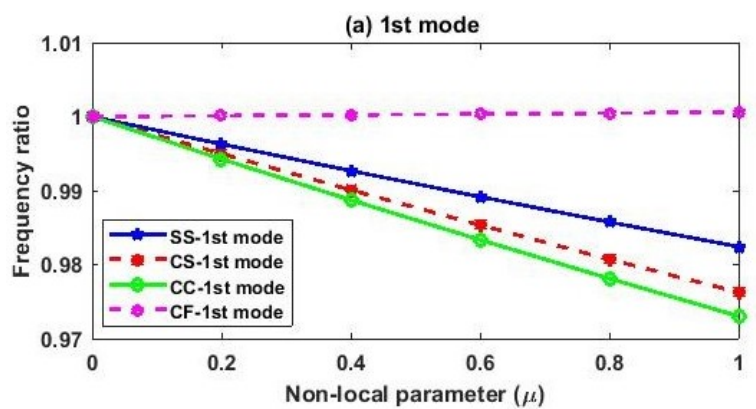

(c) 3rd mode

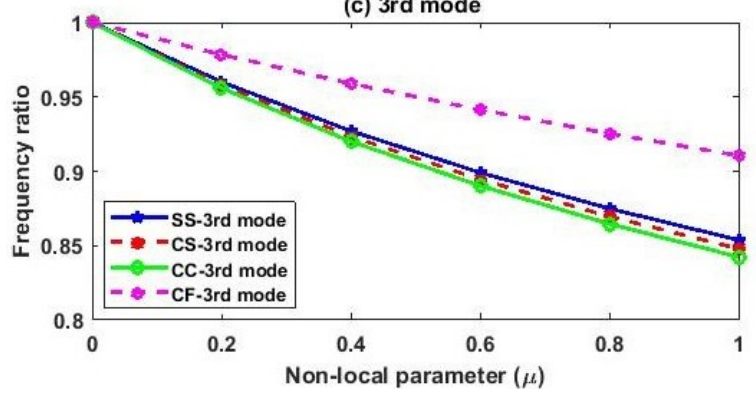

Figure 5: Variation of frequency parameter with boundary condition

Table 22: Frequency parameter $(\sqrt{\lambda})$ for SS nanobeam for different Winkler modulus parameter

\begin{tabular}{ccccc}
\hline$K$ & $\sqrt{\lambda_{1}}$ & $\sqrt{\lambda_{2}}$ & $\sqrt{\lambda_{3}}$ & $\sqrt{\lambda_{4}}$ \\
\hline 20 & 3.2176 & 6.1501 & 8.9681 & 11.5652 \\
40 & 3.3050 & 6.1631 & 8.9723 & 11.5672 \\
60 & 3.3860 & 6.1760 & 8.9765 & 11.5692 \\
80 & 3.4616 & 6.1888 & 8.9807 & 11.5711 \\
100 & 3.5325 & 6.2016 & 8.9848 & 11.5731 \\
\hline
\end{tabular}

Table 23: Frequency parameter $(\sqrt{\lambda})$ for CS nanobeam for different Winkler modulus parameter

\begin{tabular}{ccccc}
\hline$K$ & $\sqrt{\lambda_{1}}$ & $\sqrt{\lambda_{2}}$ & $\sqrt{\lambda_{3}}$ & $\sqrt{\lambda_{4}}$ \\
\hline 20 & 3.9490 & 6.9000 & 9.6884 & 12.2495 \\
40 & 3.9973 & 6.9092 & 9.6918 & 12.2511 \\
60 & 4.0440 & 6.9184 & 9.6951 & 12.2528 \\
80 & 4.0891 & 6.9275 & 9.6984 & 12.2544 \\
100 & 4.1327 & 6.9366 & 9.7017 & 12.2561 \\
\hline
\end{tabular}

quency parameters of all the classical boundary conditions such as SS, CS, CC and CF are given in Tables [2225] for different $K_{w}$. In this table, $\mu=1 \mathrm{~nm}^{2}, L=20$ and $\eta=0.5$ are taken into consideration. Graphical results are depicted in Figure 6 where variation of first four frequency parameters with $\left(K_{w}\right)$ has been shown. In this figure, $\left(K_{w}\right)$ ranges from 20 to 100 with an increment of 20.

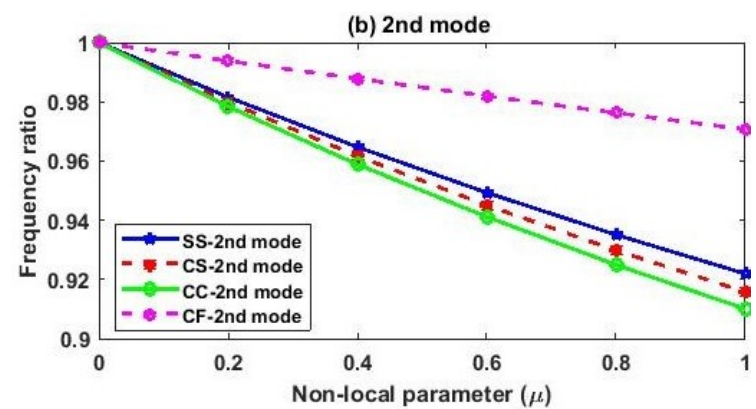

(d) 4th mode

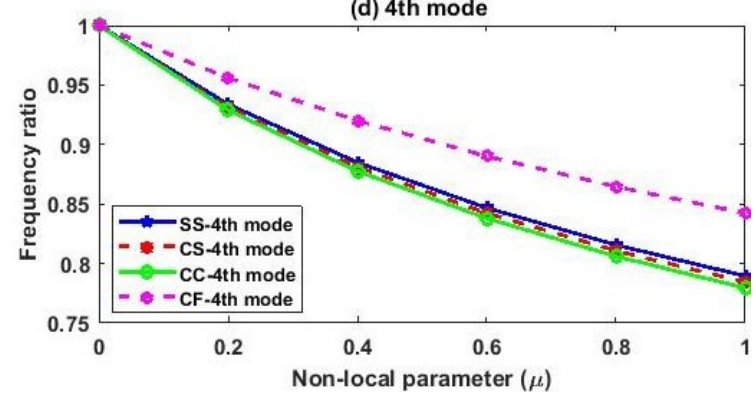

Table 24: Frequency parameter $(\sqrt{\lambda})$ for $\mathrm{CC}$ nanobeam for different Winkler modulus parameter

\begin{tabular}{ccccc}
\hline$K$ & $\sqrt{\lambda_{1}}$ & $\sqrt{\lambda_{2}}$ & $\sqrt{\lambda_{3}}$ & $\sqrt{\lambda_{4}}$ \\
\hline 20 & 4.7233 & 7.6485 & 10.4069 & 12.9311 \\
40 & 4.7518 & 7.6553 & 10.4095 & 12.9325 \\
60 & 4.7799 & 7.6620 & 10.4122 & 12.9339 \\
80 & 4.8074 & 7.6688 & 10.4149 & 12.9353 \\
100 & 4.8345 & 7.6755 & 10.4176 & 129367 \\
\hline
\end{tabular}

Table 25: Frequency parameter $(\sqrt{\lambda})$ for CF nanobeam for different Winkler modulus parameter

\begin{tabular}{ccccc}
\hline$K$ & $\sqrt{\lambda_{1}}$ & $\sqrt{\lambda_{2}}$ & $\sqrt{\lambda_{3}}$ & $\sqrt{\lambda_{4}}$ \\
\hline 20 & 2.2252 & 4.6854 & 7.6508 & 10.4067 \\
40 & 2.4604 & 4.7146 & 7.6576 & 10.4093 \\
60 & 2.6427 & 4.7433 & 7.6643 & 10.4120 \\
80 & 2.7936 & 4.7715 & 7.6711 & 10.4147 \\
100 & 2.9234 & 4.7991 & 7.6778 & 10.4174 \\
\hline
\end{tabular}

\section{Conclusions}

Vibration analysis of Single Walled Carbon Nanotube (SWCNT) resting on an exponentially varying Winkler elastic foundation is investigated by using Differential Quadrature Method (DQM). The numerical as well as graphical results are presented to show the effects of the Winkler modulus parameter, nonuniform parameter, non- 


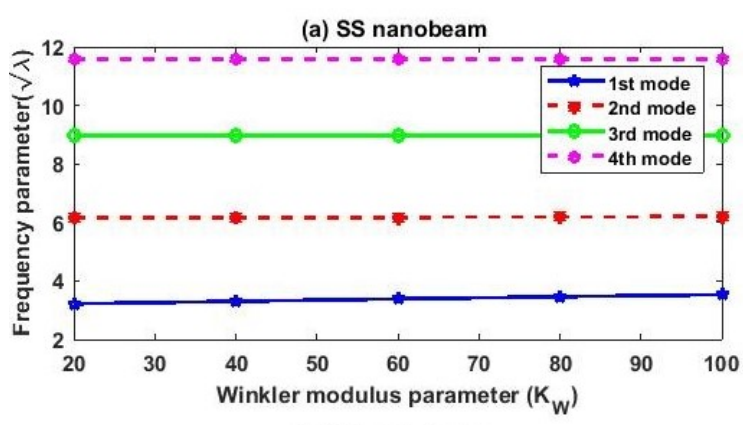

(c) CC nanobeam

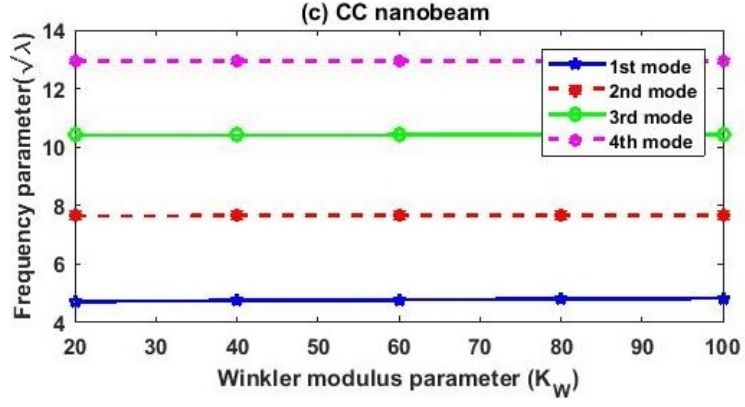

(b) CS nanobeam

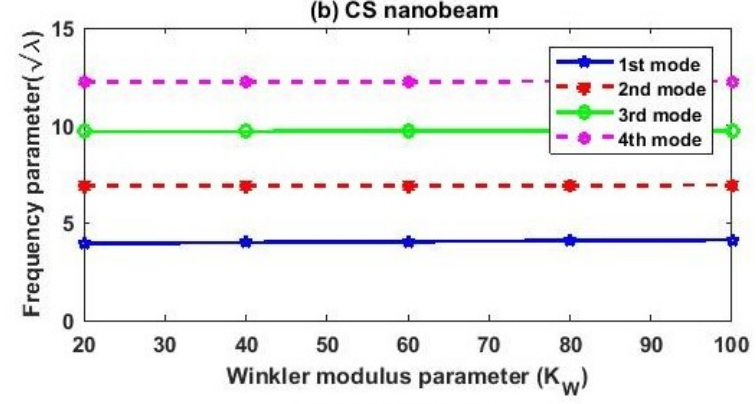

(d) CF nanobeam

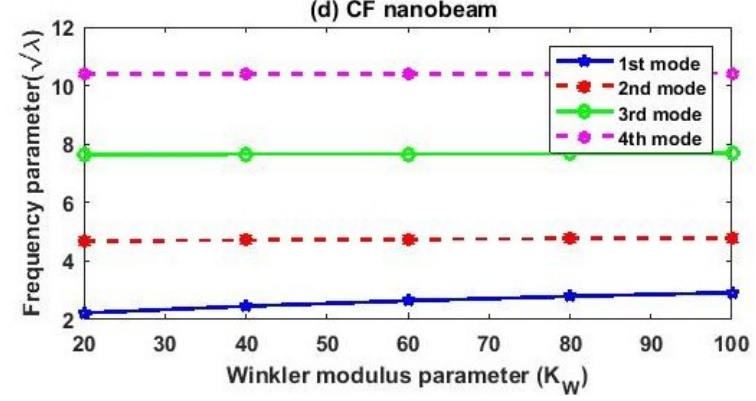

Figure 6: Variation of frequency parameter with Winkler modulus parameter

local parameter, $\mathrm{L} / \mathrm{h}$ and the boundary conditions on the frequency parameters. One may observe from the graph that the frequency parameter decreases with increase in the non-uniform parameter and this decrease is more significant in case of higher modes. It is found that clamped(CC) nanobeam possesses highest frequency parameters and cantilever(CF) nanobeam possesses lowest among all other types of boundary conditions. It is also found that the effect of nonlocal parameter is more in higher modes. One of the interesting observation is that fundamental frequency parameter of cantilever(CF) nanobeam does not decrease with increase in nonlocal parameter.

Acknowledgement: The authors are thankful to Defence Research \& Development Organization(DRDO), Ministry of Defence, New Delhi, India (Sanction Code: DG/TM/ERIPR/GIA/17-18/0129/020) for the support and funding to carry out the present research work.

\section{References}

[1] H Dai., J.H Hafner., A.G. Rinzler, D.T. Colbert, R.E. Smalley (1996) Nanotubes as nanoprobes in scanning probe microscopy, Nature, Vol-384, Issue -6605, p. 147-150.

[2] H. Peng, C. Chang, S. Aloni, T. Yuzvinsky, A. Zettl (2006) Ultrahigh frequency nanotube resonators, Phys. Rev. Lett., Vol- 97, Issue- 8, p.087203(1-4).
[3] A. Dubey, G. Sharma, C. Mavroidis, M. Tomassone, K. Nikitczuk, M. Yarmush (2004) Computational studies of viral protein nanoactuators, J. Comput. Theor. Nanosci., Vol- 1, Issue- 1, p.18-28.

[4] F Tornabene, N Fantuzzi, M Bacciocchi, E Viola (2016) Effect of agglomeration on the natural frequencies of functionally graded carbon nanotube-reinforced laminated composite doubly-curved shells, Composites Part B, Volume 89, p.187-218.

[5] N Fantuzzi, F Tornabene, M Bacciocchi, R Dimitri (2017) Free vibration analysis of arbitrarily shaped Functionally Graded Carbon Nanotube-reinforced plates, Composites Part B, Vol-115, p. 384-408.

[6] F Tornabene, N Fantuzzi, M Bacciocchi (2017) Linear static response of nanocomposite plates and shells reinforced by agglomerated carbon nanotubes, Composites Part B Vol-115, p. 449-476.

[7] D Bani'c, M Bacciocchi, F Tornabene, A. J. M. Ferreira (2017) Influence of Winkler-Pasternak Foundation on the Vibrational Behavior of Plates and Shells Reinforced by Agglomerated Carbon Nanotubes, Applied Sciences, Vol-7, p. 1-55.

[8] S. Chakraverty, L. Behera (2016) Static and Dynamic problems of nanobeams and nanoplates, First edition ,World Scientific Publishing Co.,Singapore..

[9] A.C Eringen (1972) Nonlocal polar elastic continua, Internat. J. Engrg. Sci., Vol-10, Issue- 1, p.1-16.

[10] Gorbunov-Posadov, M.I., Malikova, T.A., The design of structures on an elastic foundation, Stroiizdat, Moscow, 1973.

[11] K. Al-Hosani, S. Fadhil, A. El-Zafrany (1999) Fundamental solution and boundary element analysis of thick plates on Winkler foundation, Computers and Structures, Vol-70, p.325-336.

[12] M. HetVenyi, Beams on elastic foundations. University of Michigan Press, Ann Arbor, 1961.

[13] G. Gazetas, G. Mylonakis (1998) Seismic soil-structure interaction: new evidence and emerging Issues, Geotechnical Special Publication, Vol-75, p.1119-1174. 
[14] K.P. Soldatos, A. P. S. Selvadurai, Flexure of beams resting on hyperbolic elastic foundations. International Journal of Solids Structures 21 (1985), 373-388.

[15] J.S. Smail (1991) Large deflection response of annular plates on Pasternak foundations. International Journal of Solids Structures, Vol-27, p.1073-1084.

[16] K. K. Raju, G. V. Rao (1993) Effect of a non-linear elastic foundation on the mode shapes in stability and vibration problems of uniform columns/beams, Journal of Sound and Vibration, Vol160, p.369-371.

[17] Y. S. Shih, B. T. Blotter (1993) Non-linear vibration analysis of arbitrarily laminated thin rectangular plates on elastic foundations, Journal of Sound and Vibration, Vol-167, p.433-459.

[18] D. Zhou (1993) A General solution to vibrations of beams on variable Winkler elastic foundation, Computers \& Structures, Vol47, p.83-90.

[19] M. Eisenberger (1994) Vibration frequencies for beams on variable one- and two-paramter elastic foundations, Journal of Sound and Vibrations, Vol-176, p.577-584.

[20] L. Auersch (2008) Dynamic interaction of various beams with the underlying soil -finite and infinite, half-space and Winkler models, European Journal of Mechanics A/Solids, p.27933958.

[21] M. Eisenberger, J. Clastornik (1987) Vibrations and buckling of a beam on a variable Winkler elastic foundation, Journal of Sound and Vibration, Vol-115, p.233-241.

[22] P. Ruge, C. Birk (2007) A comparison of infinite Timoshenko and Euler-Bernoulli beam models on Winkler foundation in the frequency- and time-domain, Journal of Sound and Vibration, Vol-304, p.932-947.

[23] H.P. Lee (1998) Dynamic response of a Timoshenko beam on a Winkler foundation subjected to a moving mass, Applied Acoustics, Vol-55, p.203-215.

[24] M. H. Huang, D.P. Thambiratnam (2001) Deflection response of plate on Winkler foundation to moving accelerated loads, Engineering Structures, Vol-23, p.1134-1141.

[25] H. R. Öz, M. Pakdemirli (2006) Two-to-one internal resonances in a shallow curved beam resting on an elastic foundation, Acta Mechanica, Vol-185, p. 245-260.

[26] X. Ma, J.W. Butterworth, G.C. Clifton (2009) Static analysis of an infinite beam resting on a tensionless Pasternak foundation, European Journal of Mechanics A/Solids, Vol-28, p.697-703.

[27] O. Civalek (2007) Nonlinear analysis of thin rectangular plates on Winkler-Pasternak elastic foundations by DSC-HDQ methods, Applied Mathematical Modeling, Vol-31, p. 606-624.

[28] A Kacar, HT Tan, MO Kaya (2011) Free vibration analysis of beams on variable winkler elastic foundation by using the differential transform method, Mathematical computational applications, Vol. 16, p. 773-783.

[29] S Chakraverty and Laxmi Behera (2015) Vibration and buckling analyses of nanobeams embedded in an elastic medium, Chinese Physics B, Volume 24, p. 097305(1-8).

[30] Ö. Civalek (2004) Application of differential quadrature (DQ) and harmonic differential quadrature (HDQ) for buckling analysis of thin isotropic plates and elastic columns, Engineering Structures, Vol-26, p. 171-186.

[31] Ö. Civalek (2005) Geometrically nonlinear dynamic analysis of doubly curved isotropic shells resting on elastic foundation by a combination of harmonic differential quadrature-finite difference methods, International Journal of Pressure Vessels and
Piping, Vol-82, p. 470-479.

[32] R. Bellman, J. Casti (1971) Differential quadrature and long-term integration, J. Math. Anal. Appl., Vol- 34, Issue-2, p. 235-238.

[33] C.W. Bert, S.K. Jang, A.G. Striz (1988) Two new approximate methods for analyzing free vibration of structural components, AIAA J., Vol- 26, Issue -5, p. 612-618.

[34] S.K. Jang, C.W. Bert, A.G. Striz, (1989) Application of differential quadrature to static analysis of structural components, Internat. J. Numer. Methods Engrg., Vol-28, Issue -3, p. 561-577.

[35] C. Shu (2000) Differential Quadrature and its Application in Engineering, Springer.

[36] X. Wang, C. Bert (1993) A new approach in applying differential quadrature to static and free vibrational analyses of beams and plates, J. Sound Vib., Vol- 162, Issue -3, p. 566-572.

[37] C. Bert, X. Wang, A. Striz (1994) Static and free vibrational analysis of beams and plates by differential quadrature method, Acta Mech., Vol-102, Issue -1, p. 11-24.

[38] X. Wang, C. Bert, A. Striz, (1993) Differential quadrature analysis of deflection, buckling, and free vibration of beams and rectangular plates, Comput. \& Structures, Vol- 48 , Issue- 3, p. 473479.

[39] C.W. Bert, W. Xinwei, A.G. (1993) Striz, Differential quadrature for static and free vibration analyses of anisotropic plates, Internat. J. Solids Structures, Vol- 30, Issue-13, p. 1737-1744.

[40] C.W. Bert, M. Malik, (1996) The differential quadrature method for irregular domains and application to plate vibration, Int. J. Mech. Sci., Vol- 38, Issue- 6, p. 589-606.

[41] F Tornabene, N Fantuzzi, F Ubertini, E Viola (2015) Strong Formulation Finite Element Method Based on Differential Quadrature: A Survey, Appl. Mech. Rev, Vol- 67, Issue-2, p. 020801 (1-55).

[42] T Murmu, S Adhikari (2010) Nonlocal transverse vibration of double-nanobeam-systems, Journal of Applied Physics, Vol108, p.083514 (1-9).

[43] B. Amirian, R. Hosseini-Ara, H. Moosavi (2012) Thermal vibration analysis of carbon nanotubes embedded in two-parameter elastic foundation based on nonlocal Timoshenko's beam theory, Archives of Mechanics, Vol 64, Issue- 6, p. 581-602.

[44] J. Quan, C. Chang (1989) New insights in solving distributed system equations by the quadrature method-I. Analysis, Comput. Chem. Eng., Vol-13, Issue- 7, p. 779-788.

[45] L. Behera, S. Chakraverty (2015) Application of Differential Quadrature method in free vibration analysis of nanobeams based on various nonlocal theories, Computers \& Mathematics with Applications, Vol-69, Issue-12, Elsevier, p.1444-1462

[46] J. Reddy (2007) Nonlocal theories for bending, buckling and vibration of beams, Internat. J. Engrg. Sci., Vol-45, Issue- 2, p. 288-307.

[47] M. Aydogdu (2009) A general nonlocal beam theory: its application to nanobeam bending, buckling and vibration, Physica $E$, Vol- 41, Issue -9, p. 1651-1655.

[48] M.A. Eltaher, Amal E. Alshorbagy, F.F. Mahmoud (2013) Vibration analysis of Euler-Bernoulli nanobeams by using finite element method, Applied Mathematical Modelling, Vol-37, Issue-7, p.4787-4797. 